\title{
VIEWS AND REVIEW
}

\section{Somatosound: A review with report of 9 cases with long-term follow-up}

\author{
${ }^{1}$ Byung In Han $M D P h D,{ }^{2}$ Ho Won Lee $M D P h D,{ }^{3}$ Sanghyo Ryu $M D,{ }^{1}$ Beatrice Lucciani $M D$, \\ ${ }^{4} \mathrm{Ji}$ Man Hong $M D P h D,{ }^{5}$ Myung Cheol Bae $M D$
}

${ }^{1}$ Do Neurology Clinic, Daegu; ${ }^{2}$ Department of Neurology, Kyungpook National University, Daegu; ${ }^{3}$ Department of Neurology, Dr. Ryu's Neurology Clinic, Busan; ${ }^{4}$ Department of Neurology, Ajou University School of Medicine, Suwon; ${ }^{5}$ Bae Neurology Clinic, Yongin, Gyeonggi-do, South Korea

\begin{abstract}
Somatosound (somatic tinnitus) is associated with vascular, musculoskeletal, respiratory, or temporomandibular joint disorders. Several studies of its management have been widely reported, but only few presented long-term follow-up results. The purposes of this paper are to review the causes and management, present cases with long-term follow-up, together with previously reported cases in literatures. We treated nine patients with somatosound of vascular, hematologic, endocrinologic, muscular, and cervical origin. Follow-up were conducted routinely, and the final results were collated in 3 to 11 years. Patients with non-life-threatening causes were given counseling and palliative management. Their tinnitus becomes tolerable, gradually decreased, and even disappeared. Patients with life-threatening causes were treated immediately. Among all, there were three cases which to the best of our knowledge, are the first reported of its causes. One case was caused by a compensatory of increasing blood flow in internal carotid artery (ICA) secondary to contralateral ICA stenosis. Another had a combination of anemia and an ipsilateral jugular bulb diverticulum. The tinnitus disappeared after the anemia treated. The last was patient with hyperthyroidism. The tinnitus disappeared by controlling the condition. Even when the causes are benign and the available treatments may carry risks, the patients should not be left unmanaged. Symptomatic treatment should be given, such as counseling, sound therapy, and palliative management. Our long-term observation indicated that overall outcomes are positive when the etiologies are identified early and managed properly.
\end{abstract}

Keywords: Somatic tinnitus, somatosound, pulsatile tinnitus, vascular origin tinnitus, long-term follow-up

\section{INTRODUCTION}

Somatosound is defined as a perception of a sound which appears to be preceded or strictly linked to a somatic disorder. ${ }^{1-3}$ The terms "somatic tinnitus" and "somatosound" are used interchangeably. Its origin is usually vascular, muscular, skeletal, respiratory, or in the temporomandibular joint (TMJ). Somatosounds thus have an internal acoustic source ${ }^{4,5}$, and the source could be detected with the appropriate sensing equipment. ${ }^{4}$ The reported incidence of somatosound among all kinds of tinnitus varies, for example, $17.1 \%{ }^{6}$, $12 \%$ to $43 \%^{7}, 5 \%$ to $10 \%{ }^{8}, 16 \%$ and $83 \% .^{9}$ The variations might be explained by the differences in diagnostic criteria, lack of any agreed standards for clinical assessment ${ }^{9}$, study settings ${ }^{7}$, and its heterogeneity. ${ }^{10}$

Somatosound can be classified into vascular and non-vascular by origin ${ }^{6}$, and pulsatile and nonpulsatile according to the sound characteristics. ${ }^{11}$

Vascular tinnitus is reported to have a $4 \%$ incidence ${ }^{12}$ among all types of tinnitus. Among somatosounds, vascular tinnitus accounts for $7.6 \%$, muscular origin $4.3 \%$, and patulous eustachian tube $5.2 \%$. ${ }^{6}$ In pulsatile tinnitus, venous origin is more common than arterial. ${ }^{13}$ Anemiarelated tinnitus is infrequent and reported as $0.68 \%$ among pulsatile tinnitus. ${ }^{14}$

Among non-pulsatile tinnitus, cervicogenic somatic tinnitus (CST) arising from cervical spine disorders has a reported incidence of $43 \% .{ }^{15}$

Address correspondence to: Sanghyo Ryu, MD, Dr. Ryu's Neurology Clinic, 240, Suyeong-ro, Nam-gu, Busan 48496, South Korea. Tel: +82-51-710-8881, Email: sanghyoryu@gmail.com

Date of Submission: 12 August 2021; Date of Acceptance: 17 September 2021

https://doi.org/10.54029/2021wtk 
Pulsatile tinnitus is a tinnitus which the sound is fluctuates in synchrony with the heartbeat. ${ }^{13,16}$ It is usually unilateral but may be bilateral in systemic disease or a high cardiac output state such as anemia or hyperthyroidism, or with the presence of a midline vascular lesion. ${ }^{16,17}$

The plausible mechanisms of pulsatile tinnitus include: (1) changes in the laminar blood flow, or accelerated blood flow, resulting in audible local turbulence via the inner ear, or (2) normal blood flow sounds that are perceived more intensely due to increased bone conduction or sound conduction in the middle ear bypassing the masking effect of external sounds. ${ }^{18,19}$ Therefore, the causes of pulsatile tinnitus can be divided into vascular and non-vascular. ${ }^{20}$ Vascular causes are arterial, venous, or arteriovenous in origin. ${ }^{19,20}$ The arterial causes are atherosclerosis plaques, aneurysms of the internal carotid artery (ICA) or vascular tumors, arterial dissection, fibromuscular dysplasia, arterial atherosclerosis stenosis. Venous causes are malformations of jugular bulb (JB), transverse-sigmoid sinus aneurysm, venous hum, idiopathic intracranial hypertension, high flow through local emissary veins, or local venous stenosis. ${ }^{12,13,17-19,21}$ Arteriovenous causes include dural arteriovenous fistula (DAVF) and malformations, capillary hyperemia, and highly vascularized skull base tumors. ${ }^{18,19}$ Non-vascular causes include neoplasms like a paraganglioma, osseous pathology, Paget's disease, endolymphatic sac tumor, and systemic disease, ${ }^{17,18}$ for example, hematologic or endocrinologic disorders.

Non-pulsatile tinnitus is caused by TMJ and cervical spine (neck) disorders, ${ }^{1}$ eustachian tube dysfunction, ${ }^{4,5,11,22,23}$ or neurologic disorders such as palatomyoclonus and idiopathic stapedial muscle spasm, which are often associated with other neurologic disorders such as brain-stem tumor, infarction, or multiple sclerosis. ${ }^{24}$

Identification of the underlying cause is essential in providing appropriate treatment and determining the prognosis. The management of somatosound depends on the cause and varies from counseling, palliative, or conservative management, to aggressive intervention in lifethreatening hematologic or vascular causes.

Studies of management outcomes usually involved relatively short-term follow-up periods, with the longest follow-up being up to 3 years. This review article is based on clinical characteristics and management evaluation of our nine patients with somatosound with long-term follow-up ranged from 3 to 11 years, and selective search of the literature included review articles and case reports without restriction on date of publication. Not all types of somatosound are covered, but the common types are included. Table 1 shows the record of our nine patients' cases while Table 2 are shows some example of somatosound cases we collected from literatures.

\section{CLINICAL EXAMINATION}

Evaluation of the tinnitus patient begins with a thorough history, auscultation, otoscopy, and neurological examination. It should be determined whether the tinnitus is pulsatile or non-pulsatile, and if it is unilateral or bilateral. For vascular tinnitus, palpation, provocation, and rotation maneuvers may differentiate the origin. ${ }^{19}$ In venous tinnitus, light compression of the ipsilateral jugular vein will alleviate the tinnitus, while compression of the carotid artery has no effect. The Valsalva maneuver and rotation of the head toward the ipsilateral side will decrease the tinnitus. Contralateral venous compression, the Muller maneuver, and rotation of the head to the opposite side will increase it..$^{12,17,19,25}$ In tinnitus of arterial origin, strong compression of the carotid artery will decrease or eliminate the tinnitus. Slight compression on the ipsilateral veins, the Valsalva and Muller maneuvers, and rotation of the head toward the ipsilateral or opposite side has no effect. Slight compression on the contralateral veins also has no effect or rarely decreases the tinnitus. ${ }^{17,19}$

If there are no other abnormalities, venous tinnitus is more frequently right-sided because the right jugular vein is dominant in 70\%-80\% of cases and often triggered by physiological conditions. ${ }^{19}$

\section{PULSATILE TINNITUS}

\section{Vascular origin}

JB abnormalities, of venous origin, are commonly encountered causes of pulsatile tinnitus. JB abnormalities include: 1 ) high riding jugular bulb (HRJB), when it extends above the inferior bony annulus, if it rises to the level of the basal turn of the cochlea, or if the dome of the bulb encroaches to within $2 \mathrm{~mm}$ of the floor of the internal auditory canal; 2) jugular bulb diverticulum (JBD) which is defined as an irregular outpouching that may project into the middle ear cavity, mastoid cavity, or medially toward the petrous apex, and 3 ) dehiscent $\mathrm{JB}$, when there is a thin or absent bony septum (sigmoid plate) between the JB and middle ear space. ${ }^{12,26-28} \mathrm{JB}$ is not present at birth. ${ }^{26}$ It forming after 2 years. Prevalence 


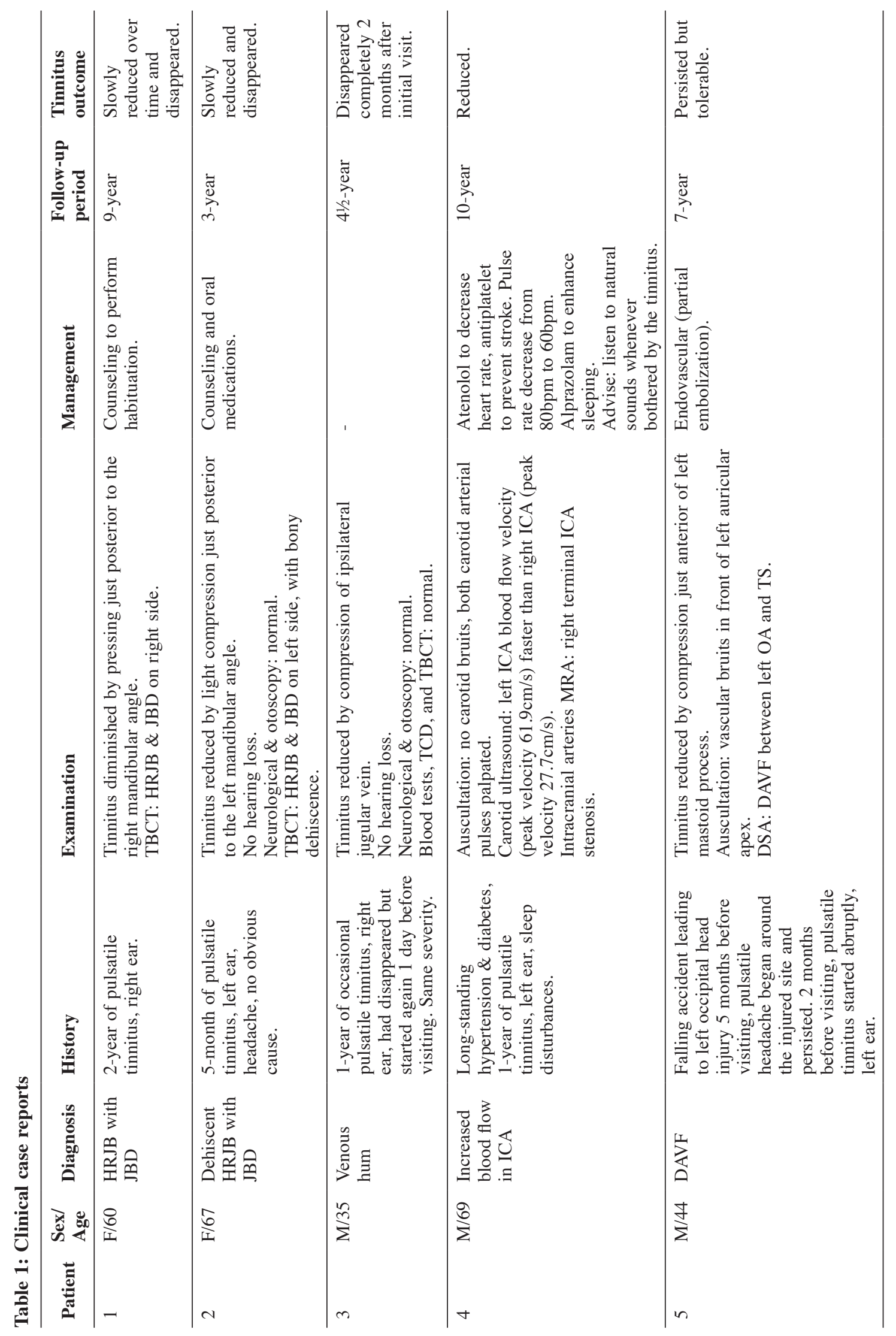




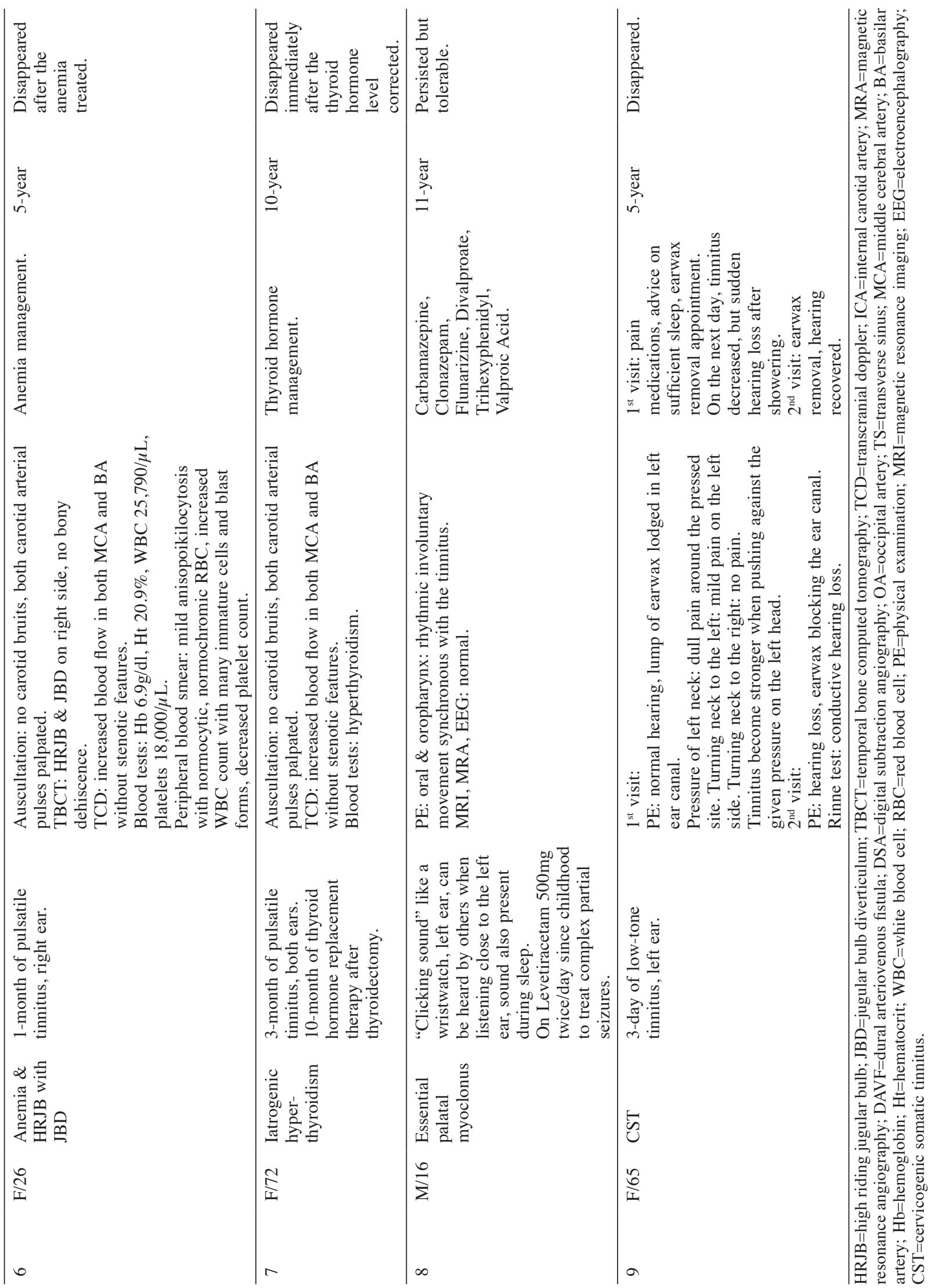




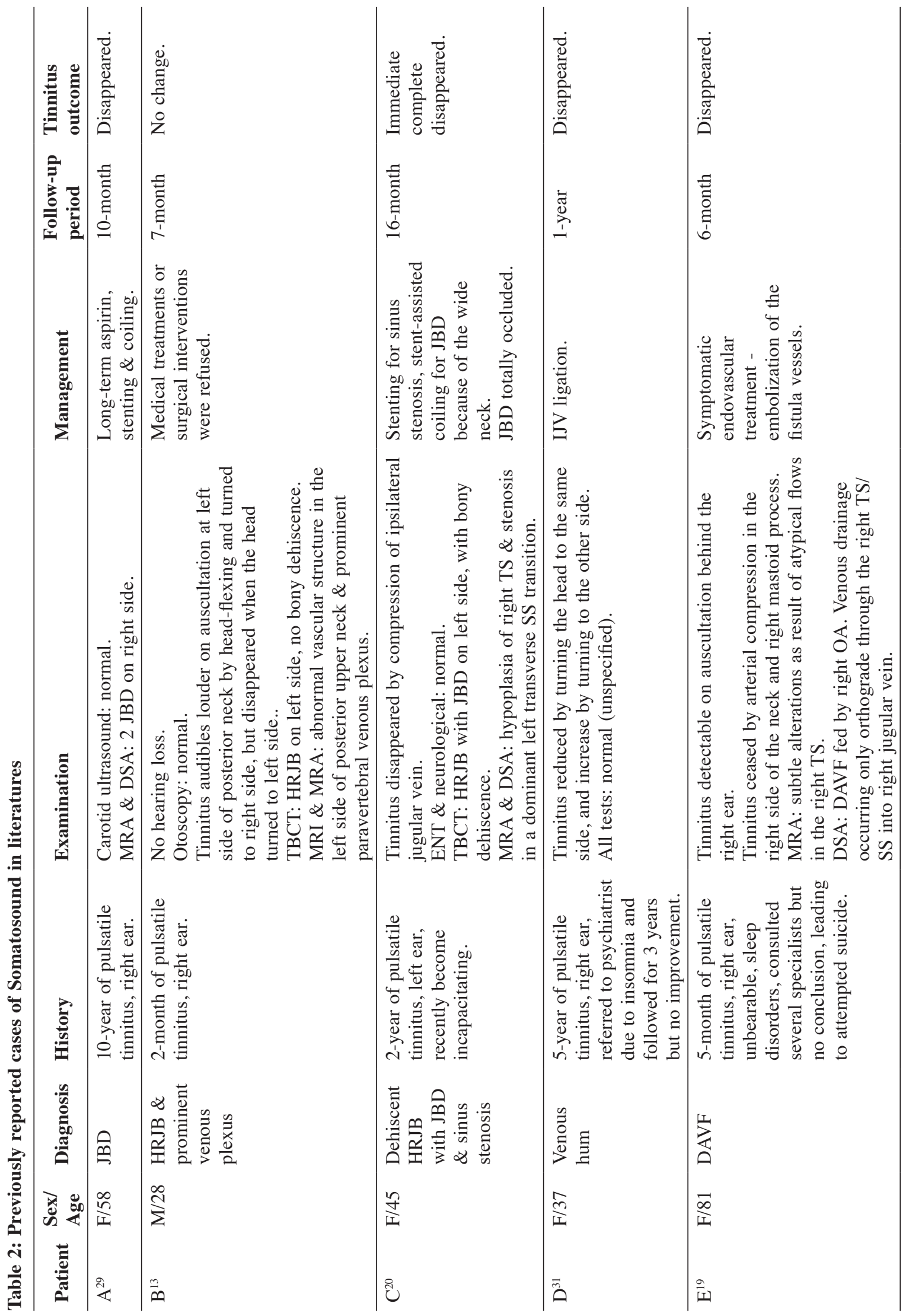




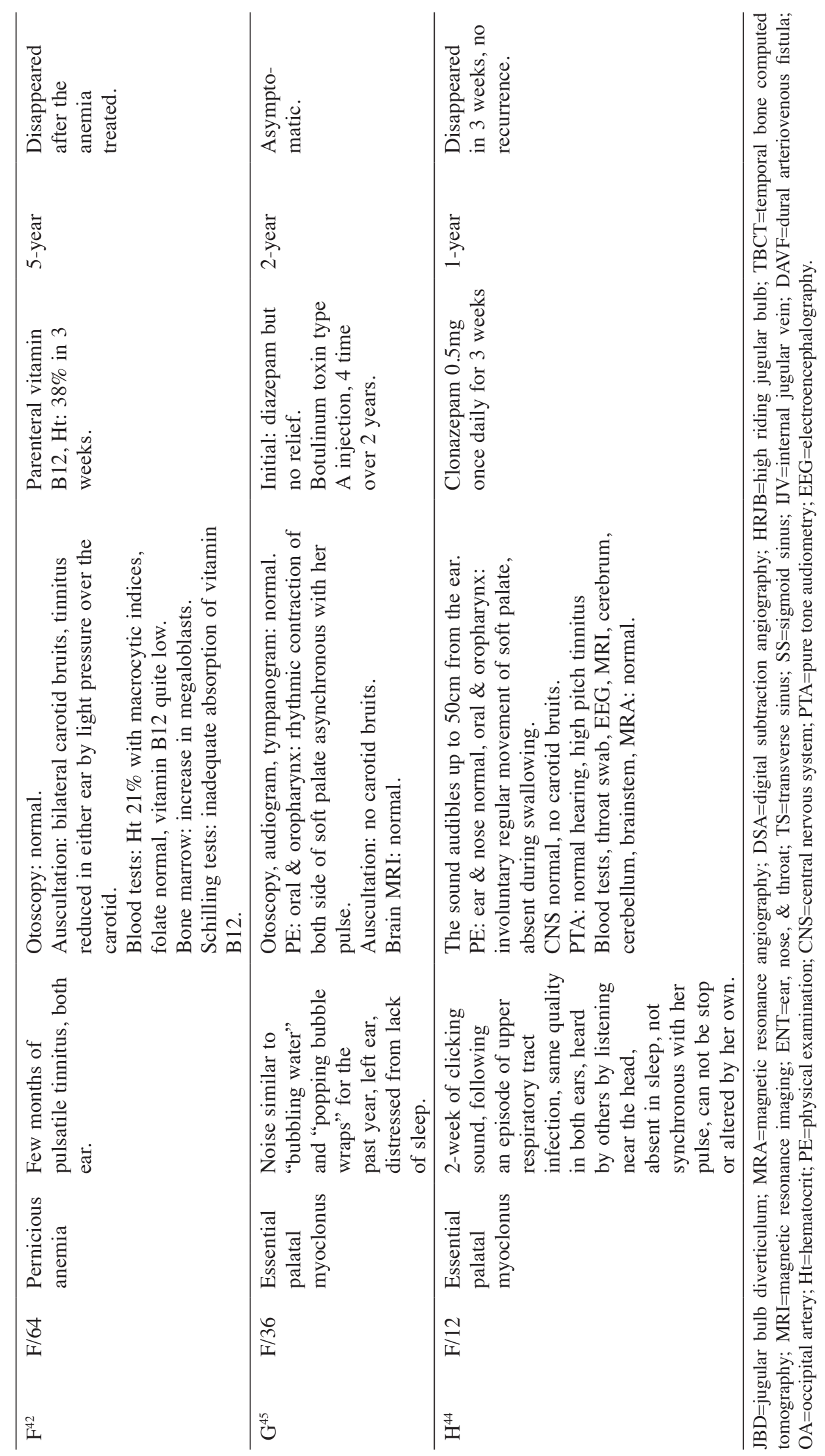


of JB abnormalities increases during the first four decades of life and stabilizes thereafter. ${ }^{29}$ The patients may be asymptomatic ${ }^{19,26,29}$, or may experience tinnitus, vertigo, hearing loss, and other symptoms which vary based on the extent, position, and the affected structures. ${ }^{26}$

For HRJB, initial treatment can be tinnitus retraining therapy, medication, or sound therapy. ${ }^{12}$ Surgical treatment should be considered only when initial treatment fails. This includes jugular vein ligation, surgical lowering, and transcatheter endovascular coil embolization. Surgery has complications such as venous flow disruption, intracranial hypertension, facial nerve injury, and conductive hearing loss. ${ }^{12,27}$

In JBD, the diverticula may cause pulsatile tinnitus through turbulent flow or by expansion into adjacent structures ${ }^{29}$ Endovascular treatment is a valid, safe method to treat the underlying cause $\mathrm{e}^{20,29}$ with total amelioration of the tinnitus. For patients who do not want an intervention, counseling is an option as long the underlying causes are not life-threatening.

For a dehiscent JB, several treatment options are mentioned including jugular vein ligation, transcatheter endovascular coil embolization, and surgical covering, reinforcement, or reconstruction. ${ }^{30}$ However, as mentioned, there are several potential complications. Thus, both outcomes and risks should be evaluated properly.

Among our nine patients with somatosound, patient 1 and 2 had JB abnormalities which were given only counseling to perform habituation and oral medications. The tinnitus had been successfully managed and slowly disappeared. The temporal bone computed tomography (TBCT) of both patients and illustration of digital compression examination are presented. (Figure $1 \mathrm{~A}-\mathrm{C}$ ) The comparative cases which we found in literatures were Patient $\mathrm{A}^{29}, \mathrm{~B}^{13}$, and $\mathrm{C}^{20}$. Patient A had JBD which treated by endovascular intervention and remains asymptomatic. Patient $B$ is the case example of patient with HRJB, presented together with prominent venous plexus, who refused any medical treatments or surgical interventions. On follow-up 7 months after admission, no symptomatic changes on the characters of his tinnitus have been noted. In this case, it might worthy to try different approach such as counseling, sound therapy or others, and follow it over a longer period of time, with the expectation to, at least, reduce the nuisance of the tinnitus. Patient $\mathrm{C}$ reported to have a dehiscent HRJB with JBD associated with transversesigmoid sinus stenosis. Considering that the
JBD had a wide neck, endovascular management was performed with total occlusion of JBD, and the patient experienced immediate complete regression of the tinnitus.

Venous hum, which is an idiopathic vascular tinnitus, thought to be caused by turbulent blood flow in the ipsilateral internal jugular vein (IJV). When a venous origin is suspected, a TBCT should be done to rule out JB and other venous anomalies. The diagnosis of a venous hum can only be made after the exclusion of other disorders. ${ }^{12}$ Venous hum is usually manageable by counseling and facilitating habituation. However, ligation of the IJV may be warranted if the venous hum is loud enough to disturb sleep or hearing. ${ }^{31,32}$ The relief of tinnitus is immediate and usually permanent. Our patient 3 presented with venous hum which the tinnitus had disappeared in 2 months completely without any intervention. Meanwhile, venous hum in patient $\mathrm{D}^{31}$ had caused her a bothering tinnitus and insomnia which she had been referred to psychiatrist but no improvement. In this situation, further intervention should be taken. Ligation of the IJV was performed and the tinnitus disappeared.

Of arterial origin, atherosclerotic plaques and stenosis are the most common cause of pulsatile tinnitus in the elderly. It accounts for approximately $8 \%-20 \%$ of all pulsatile tinnitus cases. ${ }^{33}$ The pathophysiological mechanism producing the pulsatile sound is turbulent blood flow, either secondary to stenosis or an increasedinflow. ${ }^{8}$ The tinnitus may be ipsilateral, secondary to the turbulent flow, or the closure of a vessel on one side of the body may lead to a compensatory acceleration inflow in the contralateral vessel, which then becomes symptomatic and leads to contralateral tinnitus. ${ }^{19}$ Our Patient 4 had pulsatile tinnitus originating from an increased blood flow in the ICA, (Figure 2A) which was a compensatory acceleration secondary to contralateral ICA stenosis. (Figure 2B) Even though the mechanism has been previously postulated ${ }^{19}$, no case has reported. To the authors' knowledge, ours is the first case report of this kind of somatosound.

Of arteriovenous origin, DAVF, counted as a rare cause of tinnitus, causing $2 \%-20 \%$ of pulsatile tinnitus cases. ${ }^{34}$ It is usually an acquired, abnormal connection between an artery and vein without an intervening nidus, located along the dura or within a dural sinus. ${ }^{17}$

There are 2 classification systems for DAVF. The most commonly used, the BordenShucart classification, categorizes the fistulae 

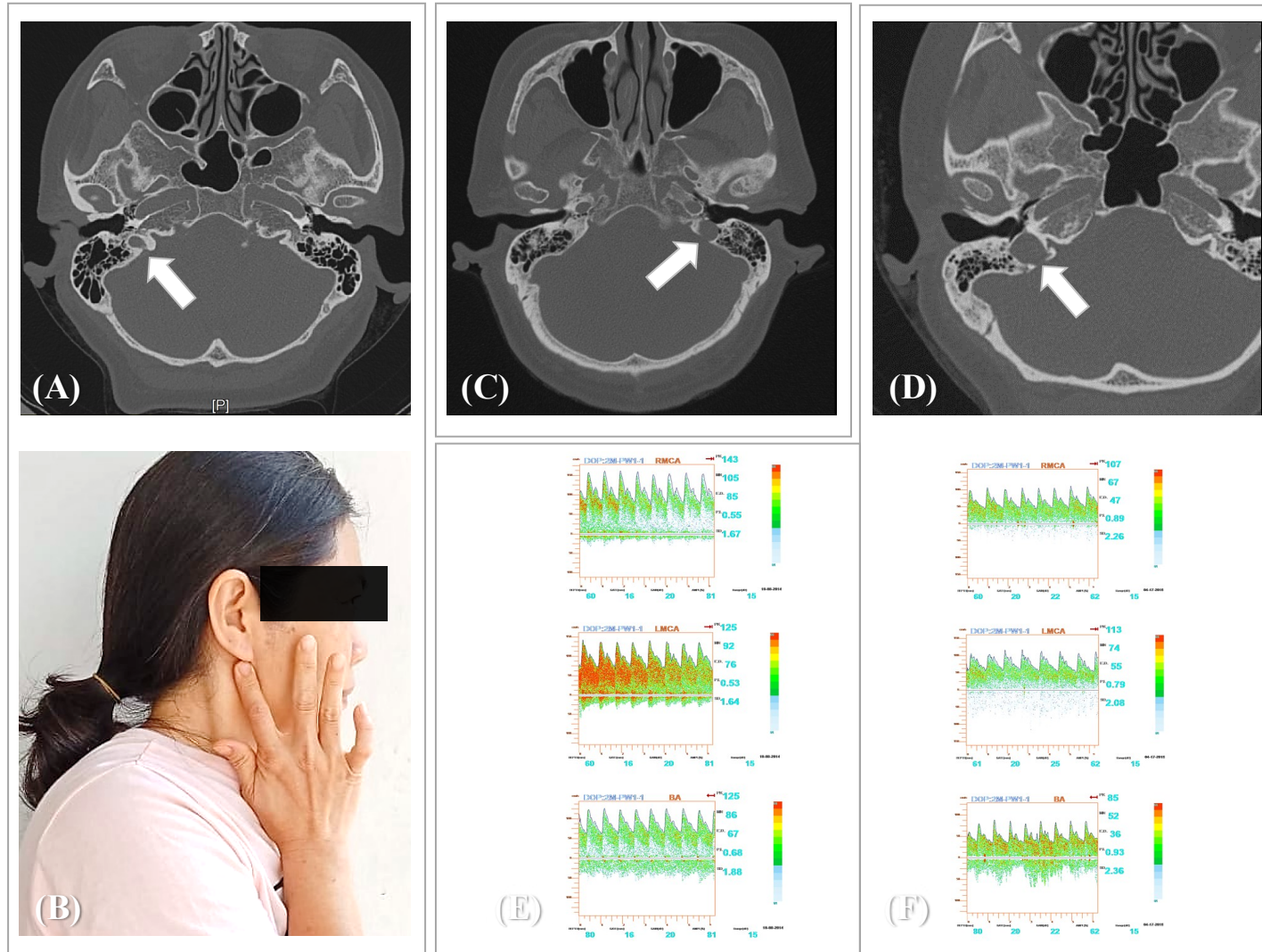

Figure 1. (A) HRJB with JBD (Patient 1). Axial image of temporal bone CT showed a HRJB with JBD at the right side (arrow). No sign of bony dehiscence. (B) An illustration picture demonstrated light digital compression of ipsilateral jugular vein which will decrease or diminish tinnitus in venous origin. (C) A dehiscent HRJB with JBD (Patient 2). Axial image of temporal bone CT showed a HRJB with JBD and bony dehiscence atthe left side (arrow). (D) Anemia and HRJB with JBD (Patient 6). Axial image of temporal bone CT showed HRJB with JBD at the right side (arrow) without bony dehiscence. (E) Patient 6. TCD test showed increased blood flows of the both middle cerebral arteries and basilar artery without stenotic features. (F) Patient 6. After remission of her leukemia, the blood flows became normalized.

into types I, II, and III, based on venous drainage characteristics. ${ }^{35,36}$ The Cognard classification divides DAVF into types I-V and creates subclassifications based on the shunt location, venous drainage characteristics, and venous outflow angioarchitecture. ${ }^{35,37}$ Detailed descriptions of the classification can be found elsewhere in the literatures..$^{35,36,37}$

Clinical manifestations of DAVF include pulsatile tinnitus, headaches, visual symptoms, and insomnia. There may be more serious symptoms, such as intracranial hemorrhage. ${ }^{8}$ Depending on the location and venous drainage pattern, DAVF arises mostly from the transverse, sigmoid, and cavernous sinuses. ${ }^{34}$ Arterial inflows are mainly from dural branches of the carotid artery, most frequently the occipital artery (OA). ${ }^{19}$ Compression of the $\mathrm{OA}$ against the mastoid process, therefore often reduces tinnitus. ${ }^{19}$
The evaluation of flow dynamics is limited on magnetic resonance imaging (MRI), magnetic resonance angiography (MRA), or conventional computed tomography angiography, therefore, digital subtraction angiography (DSA) is considered as the gold standard for detecting and evaluating DAVF. ${ }^{19}$ The current standard treatment consists of endovascular embolization and/or neurosurgical destruction when necessary. ${ }^{19,34}$

Our patient 5 had a DAVF which only be treated partially by embolization due its complex location. The tinnitus still persists but tolerable. The illustration of compression examination and DSA result are presented. (Figure $3 \mathrm{~A}-\mathrm{C}$ ) Patient $\mathrm{E}^{19}$ is a similar case to Patient 5. The tinnitus became too much affected her quality of life even led to attempted suicide. In this case, complete fistula obliteration was not indicated since the patient showed no risk of neurological complications. 

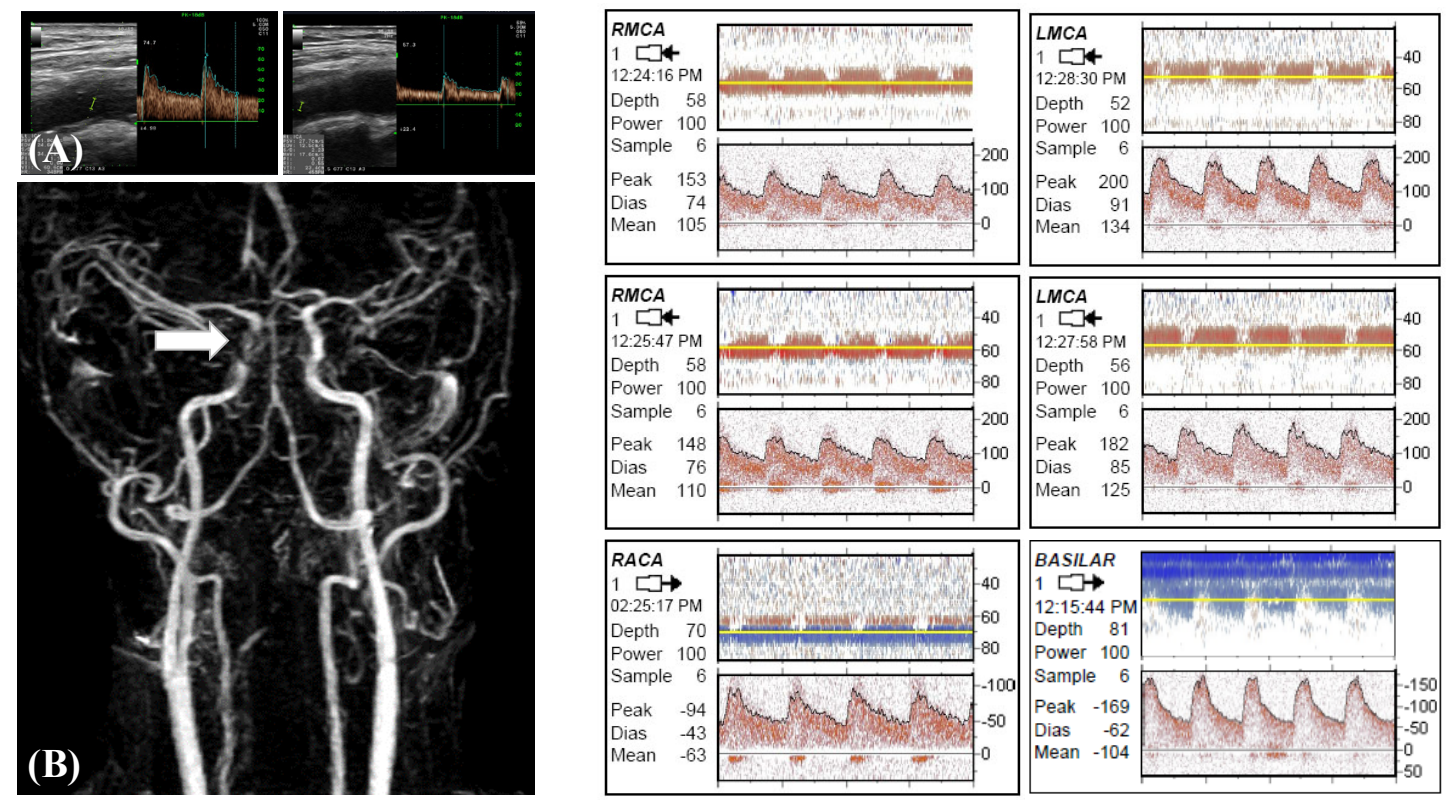

Figure 2. (A) Increased blood flow in ICA (Patient 4). Carotid ultrasound revealed that peak blood flow velocity in the left ICA $(61.9 \mathrm{~cm} / \mathrm{s})$ was faster than the right ICA $(27.7 \mathrm{~cm} / \mathrm{s})$ in the cervical area. (B) Patient 4. Stenosis in the right intracranial ICA. The MRA of his intracranial arteries showed stenosis in the right intracranial ICA (arrow). (C) Iatrogenic hyperthyroidism (Patient 7). Increased blood flows of the both middle cerebral arteries and basilar artery without stenotic features were observed by TCD exam.

Instead, only symptomatic endovascular treatment was performed, which was sufficient toresolved the unbearable tinnitus.

\section{Non-vascular origin}

Hematologic and endocrinologic conditions such as anemia and hyperthyroidism can cause hemodynamic alterations. Increased cardiac output and blood flow create turbulence, resulting in pulsatile tinnitus ${ }^{12,14,38}$, which is often bilateral. ${ }^{17,39-41}$

Our Patient 6 is a unique case in which she had both anemia and JBD, and the pulsatile tinnitus was ipsilateral to the JBD. (Figure $1 \mathrm{D}$ ) Transcranial Doppler (TCD) demonstrated increased blood flow in her initial visit (Figure 1E), which become normalized on follow-up 5 years later. (Figure 1F) Her tinnitus disappeared after recovered from the anemia following her treatment. Therefore, anemia played the key role in her pulsatile tinnitus. We can also postulate that obscured JBDs which do not present with tinnitus might be abundant. To the authors' knowledge, Patient 5 is the first case of somatosound reported with such a combination of causes and clinical course. Patient $\mathrm{F}^{42}$ had bilateral pulsatile tinnitus which concluded to be arises from her pernicious anemia condition.
The tinnitus disappeared right after her anemia resolved. For patient 7 , hyperthyroidism appeared to be the underlying cause of her bilateral pulsatile tinnitus. Increased blood flow was demonstrated in her TCD result. (Figure 2 C) Management of her hyperthyroidism resolved the tinnitus, and she remained asymptomatic during long-term follow-up. Although hyperthyroidism is widely described in the literature as a cause of tinnitus, to the authors' knowledge, our Patient 7 is also the first case report of this kind.

Considering these cases, evaluation of patients with pulsatile tinnitus therefore requires a whole blood count and thyroid function tests to be done to eliminate hemodynamic causes.

\section{NON-PULSATILE TINNITUS}

Essential palatal myoclonus, characterized by involuntary rhythmic contraction of palatal musculature as well as pharynx $x^{43-45}$, had been reported to be one of the cause for non-pulsatile tinnitus, as appeared to our Patient 8. The structures around his pharynx, including the soft palate, uvula, and the base of the tongue, showed centrifugal rhythmic involuntary movement synchronous with the tinnitus (Figure 3D) (Supplementary data-Video 1) $)^{\mathrm{a}}$, it was also visible in his submental triangle area 
(Figure 3 (E) (Supplementary data-Video 2) .

There are two types of palatal myoclonus, symptomatic and essential. Symptomatic palatal myoclonus is usually associated with hypertrophic degeneration of the inferior olivary nucleus, while essential palatal myoclonus has been described as either idiopathic, psychogenic, voluntary, central, or peripheral in origin, with no focal neurological lesions on imaging. ${ }^{44,45}$ Diagnosis is primarily made by clinical oropharynx examination. Brain MRI is needed to differentiate between the essential and symptomatic. ${ }^{44}$ The sound can be perceived unilaterally and bilaterally depends on the cases. No definite cause of essential palatal myoclonus has been determined, therefore there is no standard treatment. Several methods have been described in the literature, including anticonvulsants and botulinum toxin injections. . $^{44,45}$

Patient $\mathrm{G}^{45}$ with essential palatal myoclonus was initially treated with oral diazepam but experienced no relief. After that, she was treated with botulinum toxin type A injection which then completely relieved the tinnitus. Meanwhile, patient $\mathrm{H}^{44}$, who also diagnosed with essential palatal myoclonus, was treated with oral clonazepam $0.5 \mathrm{mg}$ once daily for 3 weeks and experienced total relieve of her tinnitus with no recurrence after 1 year.

As for the other cause of non-pulsatile tinnitus, a subgroup of patients can be defined where the tinnitus is related to the somatosensory system of the cervical spine. This is known as CST..$^{15,46}$ Clinical diagnosis of $\mathrm{CST}^{47}$ can be made when there is a history of at least one of the following before onset: (1) evident history of head or neck trauma, (2) some manipulation of the teeth, jaw, or cervical spine, (3) recurrent episodes of pain in the head, neck, or shoulder girdle, (4) temporal coincidence of the appearance or increase of pain and tinnitus, (5) an increase of tinnitus during certain postures when at rest, walking, working, or sleeping, and (6) intense bruxism during the day or night.

Other specific clinical criteria have been suggested ${ }^{46}$ : (1) neck pain, (2) impairment of cervical range of motion, in particular, rotation, (3) modulation of tinnitus by head and neck movement or posture, (4) tenderness of cervicaloccipital muscles. A positive diagnosis of CST is made when all four criteria are met.

Among all criteria, the primary diagnostic is the temporal coincidence of onset or increase of neck pain and tinnitus. ${ }^{15}$ This was the case with our Patient 9. It remains unclear if the earwax had also contributed in triggering her tinnitus.
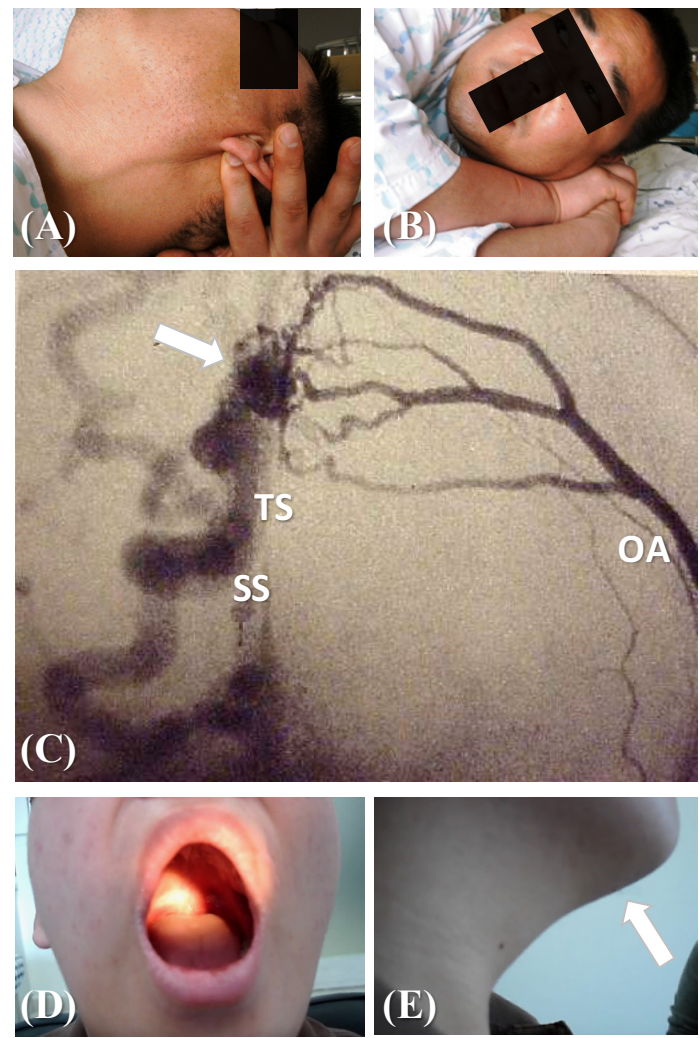

Figure 3. (A) DAVF (Patient 5). Digital compression anterior to the left mastoid process reduced the tinnitus. (B) Patient 5. Compression using knuckles together with body position gravitation against the knuckles pressure facilitated the compression to the occipital artery thusdiminished the tinnitus. (C) Patient 5. Left ECA angiography showed transverse sigmoid sinus DAVF (arrow) fed by OA. (D) Essential palatal myoclonus (Patient 8). The structure around the pharynx including soft palate, uvula, and tongue showed centrifugal rhythmic involuntary movement synchronous tothe clicking sound. The lips, larynx, and diaphragm were not involved.

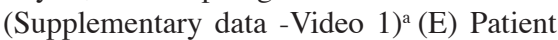
8 . The rhythmic involuntary movement also shown onthe submental triangle area (arrow). $(\text { Supplementary data -Video } 2)^{\mathrm{b}}$

${ }^{a}$ Video 1 can be seen on Do Neurology YouTube channel https://youtu.be/ZjmnFXtixgg, also can be accessed on YouTube using keywords: "palatal myoclonus AND Do neurology."

${ }^{b}$ Video 2 can be seen on Do Neurology YouTube channel https://youtu.be/cCBKa2YBT08, also can be accessed on YouTube using keywords: "palatal myoclonus AND Do neurology." 
However, the diagnosis for underlying cause as CST was made based on the increased neck pain and tinnitus on resisting applied pressure, consistent with that primary diagnostic criteria also with the clinical diagnosis criteria suggested by Sanchez et al.

Several treatments had been proposed for treating CST, including cervical physical therapies such as exercise, transcutaneous electrical nerve stimulation, manipulation of the cervical spine and trigger point deactivation by ischemic compression, steroid and lidocaine injections, mechanical treatments focused on normalizing cervical spine mobility through repetitive movements, joint mobilization, and soft tissue massage, which shown to be effectively improved the tinnitus. ${ }^{48}$

\section{CONCLUSION}

Although this case report does not cover all types of somatosound, the common types are included. Our impression based upon long-term observation is that overall outcomes are good if the etiology is determined and treated early.

Life-threatening underlying causes should be treated urgently. However, benign or nonlife-threatening causes might be left untreated, but definitely should not be neglected. Even though the causes are benign, and one might conclude that there are no risks necessarily taken to treat them, these patients should not be left unmanaged. Such patients can be offered symptomatic management. Counseling is very important to reduce the nuisance of tinnitus. Facilitating habituation with sound therapy also play a significant role. Palliative management for sleeping problems, anxiety, or decrease heart rate are options to reduce the nuisance of vascular tinnitus. Such management should be offered to relieve the patient's distress.

The management of somatosound varies depending on the causes, the options available to the therapists, and the patient's preferences. The long-term follow-up results of our patients showed that somatosound could disappear or weaken with time, often achieved through habituation imposed by the patients themselves. The authors hope that this paper will give therapists an overall understanding of somatosound management.

\section{DISCLOSURE}

Conflict of interest: None

\section{REFERENCES}

1. Ralli M, Greco A, Cialente F, et al. Somatic tinnitus. Int Tinnitus $J$ 2017;21(2):112-21.

2. Ralli M, Salvi RJ, Greco A, et al. Characteristics of somatic tinnitus patients with and without hyperacusis. PLoS ONE. 2017;12(11):e0188255.

3. Levine RA. Somatic (craniocervical) tinnitus and the dorsal cochlear nucleus hypothesis. Am J Otolaryngol 1999;20:351-62.

4. Henry JA, Zaugg TL, Myers PJ, Kendall CJ. Progressive tinnitus management: Clinical handbook for audiologists. Plural Publishing Inc.; 2010.

5. Henry JA, Roberts LE, Caspary DM, Theodoroff SM, Salvi RJ. Underlying mechanisms of tinnitus: Review and clinical implications. J Am Acad Audiol 2014;25(1):5-22.

6. Yoo HJ, Park SN, Kim DK, et al. Incidence and clinical characteristics of patients with tinnitus according to diagnostic classification. Korean $J$ Otorhinolaryngol Head Neck Surg 2011;54(6):392-8.

7. van der Wal A, Michiels S, van de Heyning P, et al. Treatment of somatosensory tinnitus: A randomized controlled trial studying the effect of orofacial treatment as part of a multidisciplinary program. $J$ Clin Med 2020;9(3):705.

8. Herraiz C, Aparicio JM. Diagnostic clues in pulsatile tinnitus (Somatosounds). Acta Otorrinolaringol 2007;58(9):426-33.

9. Michiels S, Sanchez TG, Oron Y, et al. Diagnostic criteria for somatosensory tinnitus: A delphi process and face-to-face meeting to establish consensus. Trends in Hearing 2018;22:1-10.

10. Ralli M, Greco A, Turchetta R, Altissimi G, de Vincentiis M, Cianfrone G. Somatosensory tinnitus: Current evidence and future perspectives. J Int Med Res 2017;45(3):933-47.

11. Han BI, Lee HW, Ryu S, Kim JS. Tinnitus update. J Clin Neurol 2021;17(1):1-10.

12. Bae SC, Kim DK, Yeo SW, Park SY, Park SN. Single-center 10-year experience in treating patients with vascular tinnitus: Diagnostic approaches and treatment outcomes. Clin Exp Otorhinolaryngol 2015;8(1):7-12.

13. Jung H, Kim JR, Kim SH, Song JJ. Pulsatile tinnitus related with prominent venous plexus: Case report and literature review. Korean J Otorhinolaryngol Head Neck Surg 2017;60(1):38-43.

14. Sunwoo W, Lee DY, Lee JY, et al. Characteristics of tinnitus found in anemia patients and analysis of population-based survey. Auris Nasus Larynx 2018;45(6):1152-8.

15. Michiels S, de Hertogh W, Truijen S, van de Heyning P. Cervical spine dysfunctions in patients with chronic subjective tinnitus. Otol Neurotol 2015;36(4):741-5.

16. Levine RA, Nam EC, Melcher J. Somatosensory pulsatile tinnitus syndrome: Somatic testing identifies a pulsatile tinnitus subtype that implicates the somatosensory system. Trends Amplif2008;12(3):24253.

17. Pegge SAH, Steens SCA, Kunst HPM, Meijer FJA. Pulsatile tinnitus: Differential diagnosis and radiological work-up. Curr Radiol Rep 2017;5(1).

18. Breda MS, Amorim J, Rocha J, Dias L. Vertebro- 
vertebral fistula presenting as a pulsatile tinnitus. BMJ Case Rep 2018;2018.

19. Hofmann E, Behr R, Neumann-Haefelin T, Schwager K. Pulsatile tinnitus: Imaging and differential diagnosis. Deutsches Arzteblatt Int 2013;110(26):4518 .

20. Trivelato FP, Araújo JFS, dos Santos Silva R, Rezende MTS, Ulhôa AC, Castro GD. Endovascular treatment of pulsatile tinnitus associated with transverse sigmoid sinus aneurysms and jugular bulb anomalies. Inter Neuroradiol 2015;21(4):548-51.

21. Grierson KE, Bou-Haidar P, Dumper J, Fagan PA. The assessment of pulsatile tinnitus - a systematic review of underlying pathologies and modern diagnostic approaches. Australian J Otolaryngol 2018;1:27-27.

22. Ward BK, Chao WC, Abiola G, et al. Twelvemonth outcomes of Eustachian tube procedures for management of patulous Eustachian tube dysfunction. Laryngoscope 2019;129(1):222-8.

23. Bance M, Tysome JR, Smith ME. Patulous Eustachian tube (PET), a practical overview. World J Otorhinolaryngol Head Neck Surg 2019;5(3):137-42.

24. Crummer RW, Hassan GA. Diagnostic approach to tinnitus - American Family Physician. Am Acad Family Physicians 2004;69(1):120-6. www.aafp.org/ afp.

25. Sismanis A. Pulsatile tinnitus: Contemporary assessment and management. Curr Opin Otolaryngol Head Neck Surg 2011;19(5):348-57.

26. Friedmann DR, Le BT, Pramanik BK, Lalwani AK. Clinical spectrum of patients with erosion of the inner ear by jugular bulb abnormalities. Laryngoscope 2010;120(2):365-72.

27. Koo YH, Lee JY, Lee JD, Hong HS. Dehiscent highriding jugular bulb presenting as conductive hearing loss. Medicine 2018;97(26):e11067.

28. Golueke PJ, Panetta T, Sclafani S, Varughese G. Tinnitus originating from an abnormal jugular bulb: Treatment by jugular vein ligation. J Vasc Surg 1987;6(3):248-51.

29. Mortimer AM, Harrington T, Steinfort B, Faulder K. Endovascular treatment of jugular bulb diverticula causing debilitating pulsatile tinnitus. BMJ Case Rep 2016;8(3):e11.

30. El-Begermy MA, Rabie AN. A novel surgical technique for management of tinnitus due to high dehiscent jugular bulb. Otolaryngol Head Neck Surg 2010;142(4):576-81.

31. Turki S al, Al-Kohlani H, Shabra S. Venous hum tinnitus. Int J Angiol 2003;12(1):32-3.

32. Nehru VI, Al-Khaboori M, Kishore K. Ligation of the internal jugular vein in venous hum tinnitus. $J$ Laryngol Otol 1993;107(11):1037-8.

33. Bathla G, Hegde A, Nagpal P, Agarwal A. Imaging in pulsatile tinnitus: Case based review. J Clin Imaging Sci 2020;10:84

34. Oh SJ, Chon YI, Kong SK, Goh EK. Multiple dural arteriovenous fistulas presenting as pulsatile tinnitus treated with external manual compression. J Audiol Otol 2017;21(3):156-9.

35. Reynolds MR, Lanzino G, Zipfel GJ. Intracranial dural arteriovenous fistulae. Stroke 2017;48:1424-31 .

36. Borden JA, Wu JK, Shucart WA. A proposed classification for spinal and cranial dural arteriovenous fistulous malformations and implications for treatment. J Neurosurg 1995;82:166-79.

37. Cognard C, Gobin YP, Pierot L, et al. Cerebral dural arteriovenous fistulas: Clinical and angiographic correlation with a revised classification of venous drainage. Radiology 1995;194(3):671-80.

38. Terzi S, Arslanoglu S, Demiray U, Eren E, Cancuri O. Carotid Doppler ultrasound evaluation in patients with pulsatile tinnitus. Indian J Otolaryngol 2015;67(1):43-7.

39. al Afif A, Alamoudi U, Al-Sayed AA, Bance M. Multiple Venous malformations as a cause of pulsatile tinnitus. Case Rep Otolaryngol 2020;2020:1-3.

40. Baomin L, Xiangyu C, Xinfeng L, et al. Interventional diagnosis and treatment of vasculogenic pulsatile tinnitus. J Otol 2014;9(1):7-15.

41. Lenkeit CP, al Khalili Y. Pulsatile tinnitus. In: StatPearls. StatPearls Publishing, Treasure Island (FL); 2020. https://www.ncbi.nlm.nih.gov/books/ NBK553153/?report=printable

42. Cochran JH, Kosmicki PW. Tinnitus as a presenting symptom in pernicious anemia. Ann Otol Rhinol Laryngol 1979;88(2):297-97.

43. Seidman MD, Arenberg JG, Shirwany NA. Palatal myoclonus as a cause of objective tinnitus: A report of six cases and a review of the literature. Ear Nose Throat J 1999;78(4):292-7.

44. Nisha T, Vinayakumar AR. Essential palatal myoclonus: A rare cause of objective tinnitus. Int $J$ Adv Med Health Res 2016;3(2):91-3.

45. Anis MM, Pollak N. Treatment of palatal myoclonus with botulinum toxin injection. Case Rep Otolaryngol 2013;2013(3):1-3.

46. Oostendorp RAB, Bakker I, Elvers $\mathrm{H}$, et al. Cervicogenic somatosensory tinnitus: An indication for manual therapy? Part 1: Theoretical concept. Man Ther 2016;23:120-3.

47. Sanchez TG, Rocha CB. Diagnosis and management of somatosensory tinnitus: Review article. Clinics 2011;66(6):1089-94.

48. Sajadi S, Forogh B, ZoghAli M. Cervical trigger point acupuncture for treatment of somatic tinnitus. J Acupunct Meridian Stud 2019;12(6):197-200. 